\title{
Chemotherapy-induced peripheral neuropathy - diagnosis, evolution and treatment
}

\author{
Dariusz lżycki ${ }^{1}$, Adam Andrzej Niezgoda ${ }^{2}$, Maciej Kaźmierczak ${ }^{3}$, Tomasz Piorunek ${ }^{4}$, \\ Natalia Iżycka ${ }^{5}$, Bogusława Karaszewska ${ }^{6}$, Ewa Nowak-Markwitz ${ }^{5}$ \\ ${ }^{1}$ Department of Cancer Immunology, Poznan University of Medical Sciences, Poznan, Poland \\ ${ }^{2}$ Department of Neurology, Poznan University of Medical Sciences, Poznan, Poland \\ ${ }^{3}$ Department of Hematology and Bone Marrow Transplantation, Poznan University of Medical Sciences, Poznan, Poland \\ ${ }^{4}$ Department of Pulmonology, Allergology and Pulmonological Oncology, Poznan University of Medical Sciences, Poznan, Poland \\ ${ }^{5}$ Department of Oncological Gynecology, Poznan University of Medical Sciences, Poznan, Poland \\ ${ }^{6}$ Oncological Unit, Regional Complexed Hospital, Konin, Poland
}

\begin{abstract}
Chemotherapy-induced peripheral neuropathy (CIPN) is one of the most frequent neurologic complications experienced by patients receiving antineoplastic drugs. Involvement of the peripheral nerves may have an important impact on daily activities and lead to severe impairment of the patient's quality of life (QoL). It seems to be of crucial importance to make a correct and early diagnosis of polyneuropathy and, if possible, spare the patient unnecessary suffering or loss of function. In the preceding article we have presented epidemiology, grading and pathogenesis of the toxic CIPN. The purpose of this article is to review current knowledge of diagnostic techniques, prevention and management strategies in the context of CIPN. Key words: cancer, treatment, chemotherapy, polyneuropathy
\end{abstract}

\section{DIAGNOSIS}

Since there exists no universally accepted standard for assessing CIPN, accuracy and reliability pose a major challenge due to various combinations of laboratory tests, physical evaluation, and grading systems used by oncologists.

\section{CLINICAL ASSESSMENT}

Patients are screened for pre-existing neuropathies, comorbidities and past chemotherapy treatment. A comprehensive physical exam alerts the physician of any abnormalities regarding sensory, motor, or autonomic function before proceeding with chemotherapy [1, 2]. Causes of peripheral neuropathy can be broadly subdivided into two main categories, namely, neuronopathy and axonopathy. Neuronopathy occurs rarely and is uncommon in CIPN. Symptoms evolve rapidly in a symmetric glove and stocking pattern after receiving only one or two dose administrations. Sensory and motor symptoms can arise in both upper and lower extremities from the onset, but sensory dysfunction tends to be more pronounced and appears earlier than motor deficit. The sensory impairment may be hardly reversible due to destruction of dorsal root ganglion (DRG) neurons. On the other hand, length-dependent axonopathy is characteristically associated with CIPN and is recognized by symptoms that evolve in a delayed fashion in a similar stocking-glove distribution, beginning in the lower limbs, and ascending proximally. Early presentation of symptoms commonly develops some time between the first and third cycles of chemotherapy, and is predominantly sensory in nature, although motor and autonomic dysfunction can occur during the course of the disease [2-5].

\section{Sensory symptoms}

Sensory symptoms in CIPN generally predominate at the beginning of the disease, such as hyperaesthesias, hypoaesthesias, and paresthesias $[2,4,6]$. A common clinical course begins with paraesthesias (tingling) and dysaesthe- 
sias at sites most distal to the trunk, progressing medially and symmetrically toward the trunk [3, 7]. By the time symptoms have advanced from the foot toward or past the knee level, the axons supplying the distal fingertips typically become involved and the nerve length-dependent process then begins in the upper extremities. In some cases, however, symptoms first develop in the upper limbs and are more intense than in the lower limbs. Such symptoms were reported in a study where vincristine CIPN was assessed in patients diagnosed with lymphoma [8]. Paraesthesias in a tear drop pattern on the abdomen also begin to develop as symptoms move medially and symmetrically towards the trunk [3]. Pain is often a prominent side effect of chemotherapy and may develop at any time during treatment. Severe pain is a serious problem for many patients and is often difficult to treat. It can manifest as an intense burning sensation, as intermittent pulses, or as a dull aching sensation. On occasion, patients may experience painful sensations from a stimulus that would not have previously provoked pain (allodynia). After receiving cisplatin some patients complained of pain from touching cold objects or having a cold breeze of air on the skin [4]. Patients reported experiencing myalgias, muscle cramps and aching, which are frequently exacerbated by activity [3]. For patients experiencing nociceptive sensory loss, painful symptoms may arise late in treatment and become chronic. Some patients may even experience progressing pain up to 6 months after completion of chemotherapy that is often associated with platinum analogs, this phenomenon being called coasting. Platinum compounds produce a pattern of sensory loss consistent with ganglionopathy. Lhermitte's phenomenon or a sensation of electrical discharge along the spine as a sign of myelopathy may be elicited, especially with the platinum derivatives. The hypersensitivity to pin or touch may be noted at the borders of sensory loss or as a late phenomenon in many patients. Prioprioceptive loss may result in pseudoathetosis or ataxia with severe functional impairment.

\section{Motor symptoms}

Motor symptoms in CIPN are less commonly reported than sensory symptoms and are usually milder. Rarely, they may be severe, progress to paralysis, or become life-threatening. Motor deficit begins usually in the distal lower extremities and follows the same stocking and glove distribution as doe's sensory neurotoxicity. This can result in mildly reduced strength of distal muscle groups, detectable only during clinical examination. Severe loss of function may result in weakness in dorsiflexion of the feet with subsequent foot drop. Axonal damage may result in spontaneous movements of the toes/feet or cause athetoid movements and muscle cramps in the calf, forearm and hand muscles. Deep tendon reflexes, especially at the ankle, are often reduced or lost as an early sign $[2,4]$. Patients may notice that they stumble when walking. They may also not feel steady on uneven surfaces or feel that they need to hold onto a handrail as they go up or down stairs. Other studies have found that patients with painful CIPN had more difficulty with fine motor tasks of the hands. They may also notice difficulty with writing, typing or buttoning.

\section{Autonomic symptoms}

Autonomic response to cytotoxic chemotherapy agents is relatively infrequent even though the cell bodies of both sensory and autonomic systems lie outside the blood-brain barrier (BBB) and are both equally vulnerable to damage. Sensory symptoms, on the other hand, especially pain or tingling, can be precipitated from a relatively smaller loss of neurons [3]. There are a variety of well-documented signs and symptoms of autonomic toxicity. They include orthostatic hypotension leading to syncope and anhidrosis early in the course of autonomic neuropathy, often distal to the trunk and progressing in a medial direction. Excessive sweating located in the head and neck area is also often reported. Additional signs and symptoms of autonomic toxicity include arrhythmias, dryness of the eyes and mouth, and gastrointestinal dysmotility, often demonstrated by alternating constipation and diarrhea or by early satiety from gastroparesis $[2,5]$. Patients may suffer urinary dysfunction caused by an atonic bladder, which results in overflow incontinence [9]. In men, erectile dysfunction may represent a very early autonomic symptom [3].

\section{Accessory investigations}

Neurophysiological assessment provides the most practical and convenient methods available to confirm a clinical diagnosis of CIPN. Testing helps to quantify parameters such as severity of signs/symptoms and rules out causes for neuropathy other than those induced by chemotherapy agents. These studies also help determine the scope and progression of CIPN; even so, the results may not necessarily reflect the severity of the patient's symptoms [2]. Diagnostic procedures of the peripheral nerve/muscle affections include nerve motor and sensory conduction testing, electromyography, evoked potentials and autonomic testing.

\section{Electroneuro/myography/Evoked potentials}

Electromyography (EMG) is a common name which includes electroneurography (ENG) or nerve conduction studies (NCS) and the proper needle EMG. Muscle involvement in the oncological context is rare; therefore the electrophysiological examination starts usually with NCS. Myelin disorders, rare in cancer patients are characterized by decreased conduction velocities while more often encountered axonal pathology shows reduction of sensory (sensory neural action potential, SNAP) and/or compound motor action poten- 
tial (CMAP) amplitudes. In patients affected with testicular cancer treated with cisplatin the amplitude of SNAP was reduced by $50-60 \%$ at the doses higher than $300 \mathrm{mg} / \mathrm{m}^{2}$. As dosage was increased to $400-700 \mathrm{mg} / \mathrm{m}^{2}$ the sensory conduction velocities were reduced by $10-15 \%$ - all of these being in favor of predominant axonal loss rather than myelin involvement. Somatosensory evoked potentials (SEP) revealed increased latencies of peripheral, spinal and central responses. Motor or autonomic functions were unaffected. These results showed that sensory neuronal degeneration is implicated in cisplatin CIPN [10]. Combined bedside assessments and laboratory studies are crucial for the oncologist to choose the right treatment for an individual patient.

\section{Vibration perception threshold}

A simple electronic device consisting of two alternatively vibrating rods that measures vibration perception threshold (VPT) has been employed in differentiating between patients at risk of developing low grade (0-1) CIPN and higher grade (2-3) CIPN. In this assessment performed during therapy with one of the epithelones, ixabepilone the intensity of alternative rod vibration (one on, the other off) was systematically lowered, until a threshold intensity was obtained in which the participant no longer correctly detected the vibrating rod. This technique proved even more effective in evaluating risk of CIPN, compared to electrophysiological studies, and showed promising results in diabetic neuropathy [11]. VPT has also been shown to be sensitive in vincristine-induced neuropathy. In the same study, a piezoelectric dynamometer was also used to quantify the extent of motor neuropathy by measuring the grip strength of the dominant hand. After the last dose of vincristine, grip strength in $56.3 \%$ of patients declined and was dose-dependent $[12,13]$.

\section{Nerve and skin biopsy}

Standard nerve conduction assessments, however, may not detect small fibre abnormalities [14]. Sural nerve biopsies were used to investigate large sensory and motor nerve fibre disorders [15]. They were carried out especially in patients with suspected inflammatory or inherited causes of CIPN. Skin biopsies are a new diagnostic tool that can be used for assessment of small fibre involvement in peripheral neuropathy. Cutaneous innervation consists mainly of unmyelinated fibres (A-delta and C-fibres), with myelinated fibres (A-fibres) making up to about $10 \%$ of the rest. A-delta fibres mediate cold threshold detection, while warm sensation and light touch are conveyed by C-fibres. A sample of skin is taken using skin blister or skin puncture techniques to analyze epidermal nerves. The skin puncture technique allows evaluation of dermal innervation, morphologic and ultrastuctural features of intraepidermal nerve fibres (IENFs). Evaluation of morphologic features such as dystrophic changes, tortuous and increasingly complex branches and axonal swelling of small fibres are signs of fibre degeneration and peripheral neuropathy. Additionally, the IENF density in patients with sensory neuropathy is significantly lower than in controls [14]. In an experiment involving paclitaxel and vincristine administration used to induce painful neuropathy in rats, investigators found a significant reduction of IENF compared to controls. The reduction of IENF with paclitaxel was found to be $29.3 \%$ and with vincristine $44.4 \%$. The authors concluded that paclitaxel and vincristine provoked painful neuropathies related to degeneration of IENF sensory fibres. Such a diagnostic tool may be used in the near future for evaluating clinical peripheral neuropathy in the patient population $[14,16]$.

\section{DIFFERENTIAL DIAGNOSIS}

Common symptoms may be seen in both hereditary and acquired demyelinating neuropathies. The stocking and glove distribution, paraesthesias, dysaesthesias and pain are similar in character also in CIPN, without specific symptomatology for any drug (except for paclitaxel known to cause a particularly acute pain syndrome and platinum derivatives related to cold-induced pain in throat/mouth/upper limbs) as in patients who suffer from metabolic peripheral nerve injuries, the most common of them being diabetic polyneuropathy. The onset of peripheral polyneuropathy after administration of medications is not always toxin induced, so a careful clinical examination by the physician is paramount to identifying peripheral nerve affection before any potentially neurotoxic treatment has been started. Neurologic symptoms in the oncological context must always prompt a search for tumour infiltration of the nervous system but can also be related to a general condition (cachexia), which should be considered a diagnosis of exclusion. Another possibility is a paraneoplastic neurological syndrome, e.g. paraneoplastic polyneuropathy (rare, often more pronounced in the upper limbs and frequently asymmetrical), cerebellopathy, Lambert-Eaton syndrome or limbic encephalitis, confirmed by laboratory tests detecting onconeuronal antibodies, especially anti-Hu or anti-CV2. Clinicians should bear in mind common medications such as metronidazole, misonidazole, colchicine and sulfasalazine which may also produce peripheral neuropathies [4]. Other differential diagnosis of peripheral neuropathy not related to neoplastic disease, such as concomitant inflammatory syndromes, should be considered as well.

\section{EVOLUTION}

Neuropathic peripheral symptoms usually emerge days to months after therapy is initiated but may continue after therapy is completed. Symptoms may even appear after chemotherapy treatment has been terminated [4]. Pattern and evolution of symptoms depend largely on the chemo- 
therapy agent employed and accumulated dosage. Platinum derivatives cause neurological symptoms in two distinct stages, based on differing pathophysiologic mechanisms, the first stage appearing rapidly and within hours of infusion, while second stage symptoms emerge much later. Oxaliplatin treatment, for example, induces neurotoxicity soon after infusion in more than $90 \%$ of patients. This form of neuropathy is acute and self-limited. Individuals receiving cumulative dosages of $540 \mathrm{mg} / \mathrm{m}^{2}$ or more may begin to experience chronic symptoms [17]. Another study showed clinical deterioration in $31 \%$ of patients with aggravation of peripheral neuropathy peaking between 2.5 and 5.5 months following discontinuation of cisplatin [18]. As mentioned, CIPN is dose related and develops coincidently with accumulating doses of neurotoxic agents and typically regresses after therapy is terminated with the exception of platinum and vincristine compounds [19,20]. The progression of CIPN after chemotherapy discontinuation was observed in 30\% of patients treated with vincristine. Deterioration of both subjective symptoms and vibration perception threshold 4 weeks after the last cycle of anticancer treatment was observed in $49.5 \%$ of all patients. Off-therapy persistence of neuropathy has also been reported following other micrototubule targeting agents, such as paclitaxel [21].

With taxanes, neurotoxic-induced symptoms can appear within 24-72 hours following administration of high doses, and a prolonged systematic exposure may increase neurologic presentation [22]. Patients report pain in the distal muscles of lower limbs one to two days after taxol infusion, supported by needle EMG studies showing myopathic changes. Individual cases of pure proximal weakness have been reported. Myalgia generally subsides after 4 to 7 days without any specific treatment [21, 22].

In milder cases, neuropathy is completely reversible, but this may take up to several weeks or months (persistence of neurologic deficit for more than three years should be considered irreversible). Among ovarian cancer patients treated with carboplatin/paclitaxel that experienced CIPN, $23 \%$ still had signs of residual neuropathy six months after the end of therapy [23]. In more severe cases, complete resolution is not possible and mild foot drop, decreased deep tendon reflexes or distal sensory deficits may be the residual signs. Sixty-six \% of treated patients find complete recovery $[19,24]$.

\section{TREATMENT}

More recently, new agents have been proposed that provide neuro-protective activity without interfering with function of anti-neoplastic medicines $[25,26]$. The peripheral neuropathy may be so pronounced and resistant to treatment, that the chemotherapy must be withdrawn, as in some cases of vincristine therapy [25]. Currently, the
Table 1. Treatment of chemotherapy-induced peripheral neuropathy

\section{Treatment of CIPN}

- Pain management (opioids, tricyclic antidepressants, anticonvulsants, serotonin-norepinephrine reuptake inhibitors, nonsteroid anti-inflammatory agents)

Antioxidants (alpha-lipoic acid, glutathione, vitamin E)

- Substances influencing ion channels (calcium/magnesium supplementation, pregabaline, carbamazepine)

Neuroprotectors (ginkgo biloba, glutamine)

treatment is mostly symptomatic and includes neuropathic pain management (opioids, tricyclic antidepressants, anticonvulsants, serotonin-norepinephrine reuptake inhibitors, nonsteroid anti-inflammatory agents) and nutritional supplements. Vitamin E lowers significantly both CIPN incidence and severity in patients treated with cisplatin $[11,25]$. Among pain-killers used in the management of neuropathic pain is gabapentine, the effect of which was noted in cancer patients with secondary myalgias receiving taxanes [26]. However, in our own experience effects if this drug are often disappointing as it requires very high doses with severe side-effects. No efficacy of gabapentin was also reported by Rao et al. [27]. An alternative to gabapentin and a more promising agent seems to be pregabaline - an antiepileptic drug known as a $a 2-\delta$ calcium channel ligand which could be recommended as a first choice drug in neuropathic pain treatment, already confirmed in diabetic painful polyneuropathy [28].

There are a few substances for treatment of CIPN being currently investigated [26]. Some of them have proven to be effective in the treatment of diabetic neuropathy. Among the most promising are those which influence cellular metabolism and act as antioxidants, including alpha-lipoic acid, glutathione, and vitamin E (Table 1). Alpha-lipoic acid was reported to bring relief to 8 out of 14 patients experiencing docetaxel plus cisplatin-related neuropathy, who were affected with gastric cancer, non-small cell lung cancer (NSCLC), and head and neck tumors [29]. Substances which interact with ion channels and prevent or diminish the incidence of CIPN appear to be effective in platinum-induced peripheral neuropathy (Table 2). These include calcium/magnesium supplementation and carbamazepine, although the latter in our experience is relatively ineffective and associated with pharmacologically induced hyponatraemia in $10 \%$ of patients (Table 3 ) [30]. Finally, ginkgo biloba, and glutamine appear to confer protection with regard to the peripheral nervous system, although the involved mechanism is not fully understood. A significant reduction in CIPN incidence, regardless of grade, was reported in colorectal cancer patients treated with oxaliplatin, while receiving glutamine. The result was not only reflected in the neurotoxicity scale, but in an assessment of quality of life 
Table 2. Substances studied as treatment modalities in chemotherapy-induced peripheral neuropathy. Antidepressants, however, may be useful in the treatment of secondary to cancer depressive syndromes. Some of the agents proved to be ineffective in CIPN are listed in Table 3

\begin{tabular}{|c|c|c|c|c|c|}
\hline Treatment & Dose & $\begin{array}{c}\text { Number of } \\
\text { patients }\end{array}$ & Type of trial & Comments & Reference \\
\hline $\begin{array}{l}\text { Calcium/magnesium } \\
\text { infusions }\end{array}$ & $\begin{array}{l}1 \mathrm{~g} \text { i.v. of each before and after } \\
\text { oxaliplatin infusion }\end{array}$ & 102 & $\begin{array}{l}\text { Double blind } \\
\text { randomized with } \\
\text { placebo }\end{array}$ & $\begin{array}{l}\text { Decreased incidence of grade } \\
2 \text { neuropathy or greater in } \mathrm{NCI} C \mathrm{CTC}\end{array}$ & {$[30]$} \\
\hline \multirow[b]{2}{*}{ Vitamin E } & $\begin{array}{l}300 \mathrm{mg} / \mathrm{d} \text { before and } \\
3 \text { months after cisplatin } \\
\text { chemotherapy }\end{array}$ & 27 & $\begin{array}{l}\text { Open label, } \\
\text { cisplatin }\end{array}$ & $\begin{array}{l}\text { CIPN in } 31 \% \text { patients with vitamin } E \\
\text { versus } 86 \% \text { without }(p<0.01)\end{array}$ & {$[25]$} \\
\hline & $400 \mathrm{mg} / \mathrm{d}$ & 81 & $\begin{array}{l}\text { Double blind, } \\
\text { randomized } \\
\text { placebo-controlled }\end{array}$ & $\begin{array}{l}\text { Interim analysis of first } \\
50 \text { patients having received } \\
\text { cisplatin }>300 \mathrm{mg} / \mathrm{m}^{2} \text { median CIPN score } \\
\text { lower in the vitamin E group }(p<0.05)\end{array}$ & [31] \\
\hline \multirow[t]{2}{*}{ Glutamine } & $\begin{array}{l}10 \mathrm{~g} \text { p.o. for } 4 \text { days, first dose } \\
24 \mathrm{~h} \text { after the completion } \\
\text { of paclitaxel, ( } 33 \text { patients } \\
\text { got glutamine, } 12 \text { had no } \\
\text { glutamine treatment) }\end{array}$ & 33 & Open label & $\begin{array}{l}\text { Reduction in the severity of neuropathy } \\
(p<0.05) \text {, weakness }(p<0,04) \text { gait } \\
\text { deterioration }(p<0,02)\end{array}$ & {$[20]$} \\
\hline & $\begin{array}{l}15 \mathrm{~g} \text { p.o. twice/d for } 7 \text { days } \\
\text { every } 2 \text { weeks following } \\
\text { oxaliplatin infusion }\end{array}$ & & & $\begin{array}{l}\text { Less grade } 1-2(17 \% \text { vs. } 39 \%) \text { and grade } \\
3-4 \text { CIPN after } 4 \text { cycles ( } 5 \% \text { vs. } 18 \%) \text { and } \\
\text { six cycles }(12 \% \text { vs. } 32 \%)\end{array}$ & {$[19,32]$} \\
\hline Alpha-lipoic acid & $\begin{array}{l}600 \text { mg i.v. once a week } \\
\text { for } 3-5 \text { weeks followed } \\
\text { by } 1800 \text { mg td p.o. until } \\
\text { significant recovery from CIPN, } \\
\text { but no longer than } 6 \text { months }\end{array}$ & 14 & Open label & $\begin{array}{l}\text { Gastric cancer, NSCLC } \\
\text { or head and neck tumors, improvement } \\
\text { in } 8 \text { patients, no response in } 6\end{array}$ & [29] \\
\hline
\end{tabular}

Table 3. Drugs ineffective in the treatment of CIPN

\begin{tabular}{|l|l|c|l|l|c|}
\hline Treatment & \multicolumn{1}{|c|}{ Dose } & $\begin{array}{c}\text { Number of } \\
\text { patients }\end{array}$ & Type of trial & Comments \\
\hline Lamotrigine & & 131 & Placebo-controlled; double-blinded & No CIPN benefit \\
\hline Gabapentin & $2700 \mathrm{mg} /$ (target dose) & 115 & Placebo-controlled; double-blinded & No CIPN benefit \\
\hline Amitriptyline & $\begin{array}{l}10 \mathrm{mg} / \mathrm{d} \text { up to } 50 \mathrm{mg} / \mathrm{d} \\
\text { then stable dose for } 4 \text { weeks }\end{array}$ & 44 & Placebo-controlled; double-blinded & No CIPN benefit \\
\hline
\end{tabular}

and frequency of chemotherapy dose reduction. Glutamine administration also proved effective in lowering severity of sensory, and incidence of motor symptoms as well as in decreased interference with daily activities in neuropathy induced by paclitaxel in breast cancer patients [26]. It is suspected that glutamine increases the nerve growth factor level that contributes to decreased rate of neural degeneration. Since some of the above studies lacked a placebo group, while other were limited to a small number of patients, there remains a need for strong evidence supporting the CIPN-counteracting effect of these substances. At present, the reduction of chemotherapy (longer neurotoxic drug-free periods) or its withdrawal is regarded as the most efficient effective method of managing CIPN.

\section{CONCLUSIONS}

Advances in early cancer detection as well as in carcinogen/anti-cancer drug metabolism understanding including molecular mechanisms of genetic control of responsiveness and predisposition to adverse effects are needed to select patients who have a better prognosis for a given type of treatment. Further studies providing this type of information will be crucial for tailoring the antineoplastic therapy in a rational way.

\section{REFERENCES}

1. Argyriou AA, Polychronopoulos P, Iconomou G, Koutras A, Kalofonos HP, Chroni E. Paclitaxel plus carboplatin-induced peripheral neuropathy. A prospective clinical and electrophysiological study in patients suffering from solid malignancies. J Neurol. 2005, 252, 1459-1464.

2. Ocean AJ, Vahdat LT. Chemotherapy-induced peripheral neuropathy: pathogenesis and emerging therapies. Support Care Cancer. 2004, 12, 619-625.

3. Stillman M, Cata JP. Management of chemotherapy-induced peripheral neuropathy. Curr Pain Headache Rep. 2006, 10, 279-287.

4. Hausheer FH, Schilsky RL, Bain S, Berghorn EJ, Lieberman F. Diagnosis, management, and evaluation of chemotherapy-induced peripheral neuropathy. Semin Oncol. 2006, 33, 15-49.

5. QuasthoffS, Hartung HP. Chemotherapy-induced peripheral neuropathy. J Neurol. 2002, 249, 9-17. 
6. Hilkens PHE, Verweij J, Stoter G, Vecht ChJ, van Putten WLJ, van den Bent MJ. Peripheral neurotoxicity induced by docetaxel. Neurology. 1996, 46, 104-108.

7. LoMonaco M, Milone M, Batocchi AP, Padua L, Restuccia D, Tonali P. Cisplatin neuropathy: clinical course and neurophysiological findings. J Neurol. 1992, 239, 199-204.

8. Höftberger R, Rosenfeld MR, Dalmau J. Update on neurological paraneoplastic syndromes. Curr Opin Oncol. 2015, 27, 489-495.

9. Shah-Khan F, Shah P. Loss of bladder sensation following taxane therapy. Chemotherapy. 2008, 54, 425-426.

10. Krarup-Hansen A, Helweg-Larsen S, Schmalbruch H, Rorth $M$, Krarup C. Neuronal involvement in cisplatin neuropathy: prospective clinical and neurophysiological studies. Brain. 2007, 130, 1076-1088.

11. Landowski LM, Dyck PJ, Engelstad J, Taylor BV. Axonopathy in peripheral neuropathies: mechanisms and therapeutic approaches for regeneration. J Chem Neuroanat. 2016, S0891-0618(16)30070-9.

12. Coppini DV, Wellmer A, Weng C, Anand P, Sonksen PH. The natural history of diabetic peripheral neuropathy determined by a 12 year prospective study using vibration perception thresholds. J Clin Neurosci. 2001, 8, 520-524.

13. Jurado J, Ybarra J, Pou JM. Isolated use of vibration perception thresholds and Semmes-Weinstein monofilament in diagnosing diabetic polyneuropathy: the North Catalonia Diabetes Study. Nurse Clin North Am. 2007, 42, 59-66.

14. Ebenezer GJ, Hauer P, Gibbons C, McArthur JC, Polydefkis M. Assessment of epidermal nerve fibers: a new diagnostic and predictive tool for peripheral neuropathies. J Neuropath Expt Neurol. 2007, 66, 1059-1073.

15. Sahenk Z, Barohn R, New P, Mendell JR. Taxol neuropathy: electrodiagnostic and sural nerve biopsy findings. Arch Neurol. 1994, 51, 726-729.

16. Siau C, Xiao W, Bennett GJ. Paclitaxel- and vincristine-evoked painful peripheral neuropathies: loss of epidermal innervation and activation of Langerhans cells. Exp Neurol. 2006, 201, 507Y14.

17. Cersosimo RJ. Oxaliplatin-associated neuropathy: a review. Ann Pharmacother. 2005, 39, 128-135.

18. Siegal T, Haim N. Cisplatin-induced peripheral neuropathy. Frequent off-therapy deterioration, demyelinating syndromes, and muscle cramps. Cancer. 1990, 66, 1117-1123.

19. Brewer JR, Morrison G, Dolan ME, Fleming GF. Chemotherapy-induced peripheral neuropathy: current status and progress. Gynecol Oncol. 2016, 140, 176-183.

20. Marmiroli P, Cavaletti G. Drugs for the treatment of peripheral neuropathies. Expert Opin Pharmacother. 2015, 5, 1-14.
21. Mielke S, Sparreboom A, Steinberg SM, [et al.]. Association of paclitaxel pharmacokinetics with the development of peripheral neuropathy in patients with advanced cancer. Clin Cancer Res. 2005, 11, 4843-4850.

22. Serkies K, Jassem J. Mięsakorak trzonu macicy. Ginekol Pol. 2012, 83, 609-612.

23. Pignata S, DePlacido S, Biamonte R, [et al.]. Residual neurotoxicity in ovarian cancer patients in clinical remission after first-line chemotherapy with carboplatin and paclitaxel: The Multicenter Italian Trial in Ovarian cancer (MITO-4) retrospective study. BMC Cancer. 2006, 6, 5.

24. Armstrong T, Almadrones $L$, Gilbert MR. Chemotherapy-induced peripheral neuropathy. Oncol Nurs Forum. 2005, 32, 305-311.

25. Pace A, Savarese A, Picardo M, [et al.]. Neuroprotective effect of vitamin E supplementation in patients treated with cisplatin chemotherapy. J Clin Oncol. 2003, 21, 927-931.

26. Marmiroli P, Cavaletti G. Drugs for the treatment of peripheral neuropathies. Expert Opin Pharmacother. 2015, 5, 1-14.

27. Rao RD, Michalak JC, Sloan JA, [et al.]. Efficacy of gabapentin in the management of chemotherapy-induced peripheral neuropathy: a phase 3 randomized double-blind, placebo-controlled, crossover trial (NOOC3). Cancer. 2007, 110, 2110-2118.

28. Rosenstock J, Tuchman M, LaMoreaux L, Sharma U. Pregabalin for the treatment of painful diabetic peripheral neuropathy: a double-blind, placebo-controlled trial. Pain. 2004, 110, 628-638.

29. Gedlicka C, Kornek GV, Scmid K, Scheithauer W. Amelioration of docetaxel/cisplatin induced polyneuropathy by alphalipoic acid. Ann Oncol. 2003, 14, 339-340.

30. Grothey A, Nikcevich DA, Sloan JA, [et al.]. Oxaliplatin-induced sensory neurotoxicity. J Clin Oncol. 2011, 29, 421-427.

31. Pace $A$, Carpano $S$, Galiè $E$, [et al.]. Vitamin $E$ in the neuroprotection of cisplatin induced peripheral neurotoxicity and ototoxicity. J Clin Oncol. 2007, 25 (18S).

32. Paré $L$, Marcuello $E$, Alte $A$, [et al.]. Pharmacogenetic prediction of clinical outcome in advanced colorectal cancer patients receiving oxaliplatin/5-fluorouracil as first-line chemotherapy. Br J Cancer. 2008, 99, 1050-1055.

33. Rao RD, Flynn PJ, Sloan JA, [et al.]. Efficacy of lamotrigine in the management of chemotherapy-induced peripheral neuropathy: a phase 3 randomized, double-blind, placebo-controlled trial, N01C3. Cancer. 2008, 112, 2802-2808.

34. van Deventer $\mathrm{H}$, Bernard S. Use of gabapentin to treat taxane-induced myalgias. J Clin Oncol. 1999, 17, 434-435. 